\title{
The Process of Solving Complex Problems
}

\author{
Andreas Fischer, Samuel Greiff, and Joachim Funke
}

\section{Acknowledgements}

This research was funded by a grant of the BMBF (FKZ: 01JG1062). We gratefully thank Sascha Wüstenberg and Daniel Holt for their comments.

Correspondence concerning this paper should be addressed to Samuel Greiff, Department of Psychology, Heidelberg University, Hauptstraße 47-51, D-69117 Heidelberg, Germany. Phone: +49 6221 547613. Fax: +49 547273. E-mail:

Samuel.Greiff@psychologie.Uni-Heidelberg.de

\begin{abstract}
This article is about Complex Problem Solving (CPS), its history in a variety of research domains (e.g., human problem solving, expertise, decision making, and intelligence), a formal definition and a process theory of CPS applicable to the interdisciplinary field. CPS is portrayed as (a) knowledge acquisition and (b) knowledge application concerning the goal-oriented control of systems that contain many highly interrelated elements (i.e., complex systems). The impact of implicit and explicit knowledge as well as systematic strategy selection on the solution process are discussed, emphasizing the importance of (1) information generation (due to the initial intransparency of the situation), (2) information reduction (due to the overcharging complexity of the problem's structure), (3) model building (due to the interconnectedness of the variables), (4) dynamic decision making (due to the eigendynamics of the system), and (5) evaluation (due to many, interfering and/or ill-defined goals).
\end{abstract}

\section{Keywords}

Complex Problem Solving, CPS, Operative Intelligence, Dynamic Problem Solving, Dynamic Decision Making, Expertise

\footnotetext{
${ }^{1}$ University of Heidelberg, Germany
} 


\section{Introduction}

In times of increasing globalization and technological advances, many problems humans have to face in everyday life are quite complex, involving multiple goals as well as many possible actions that could be considered, each associated with several different and uncertain consequences, in environments that may change dynamically and independent of the problem solvers' actions (Funke, 2003). In order to solve complex problems, people usually have to acquire and to apply knowledge about complex systems concerning the systems' structure and dynamics (Funke, 2001). Examples for Complex Problem Solving (CPS) are easily found, e.g., using unknown complex technical devices (like a new mobile phone, a computer, a vending machine, etc.), managing complex organizations (like corporations or communities) or making predictions in complex environments (like forecasts of the weather, political elections or the stock market, etc.). In research on human problem solving CPS is a matter of interest since the 1970s, when there was a shift of emphasis from simple, static, well-defined and academic problems (like the Tower of Hanoi or items of classical intelligence tests), to more complex, dynamic, ill-defined, and realistic problems (Wenke, Frensch, \& Funke, 2005). Since then, research on human problem solving focused on interviewing experts of certain knowledge domains, on studying the effects of expertise on problem solving activities and decision making, or on simulating complex problems ${ }^{1}$ based on real systems humans could have to deal with in their daily lives (like planning a day, managing an organization, fire fighting, and so on). Along with more complexity in research on problem solving new questions arose: How does expertise and prior knowledge influence problem solving in complex situations? Are there certain strategies especially useful for coping with complex problems? How is a complex situation represented in the human mind with its restricted capabilities? Which facets of intelligence are most important for solving complex problems? Some of these questions were addressed by different fields of research (e.g., research on problem solving, on expertise, on information reduction, on decision making, and research on intelligence), but in spite of a lot of fruitful research on CPS in these areas, up to now most of this research has been conducted with a focus on empirical data mining rather than theoretical considerations (see Funke, 2010), without a clear-cut definition (see Quesada, Kintsch, \& Gomez, 2005) commonly accepted in the scientific community.

The article at hand wants to contribute to the solution of this shortcoming: After summarizing the most important empirical and theoretical contributions to the field, we want to come up with a process theory of CPS based on a formal definition, applicable to the interdisciplinary field. We want to consider (a) what is known about the most im-

${ }^{1}$ Osman (2010) refers to the complex scenarios of this kind as "Complex Dynamic Control Tasks" and points out that these tasks are known in the fields of CPS, dynamic decision making, naturalistic decision making, and process control amongst others. 
portant determinants of the process of CPS in the different domains of research (such as expertise, decision making, and intelligence) and (b) how these contributions fit together if viewed under an integrative perspective.

\section{What is meant by Complex Problem Solving?}

Research on CPS produced a lot of characterizations and operationalizations of complex problems (for an overview see Frensch \& Funke, 1995), but up to now there has not been a definition of complex problems commonly accepted in the scientific community (Quesada et al., 2005). There is an ongoing debate about (a) what should be considered complex in CPS and (b) how complexity might be measured in detail (see Quesada et al., 2005 for a discussion).

The definition of CPS proposed and applied in this article is based on the constitutive concepts "complexity", "problem", and "problem solving" which in turn are understood as follows:

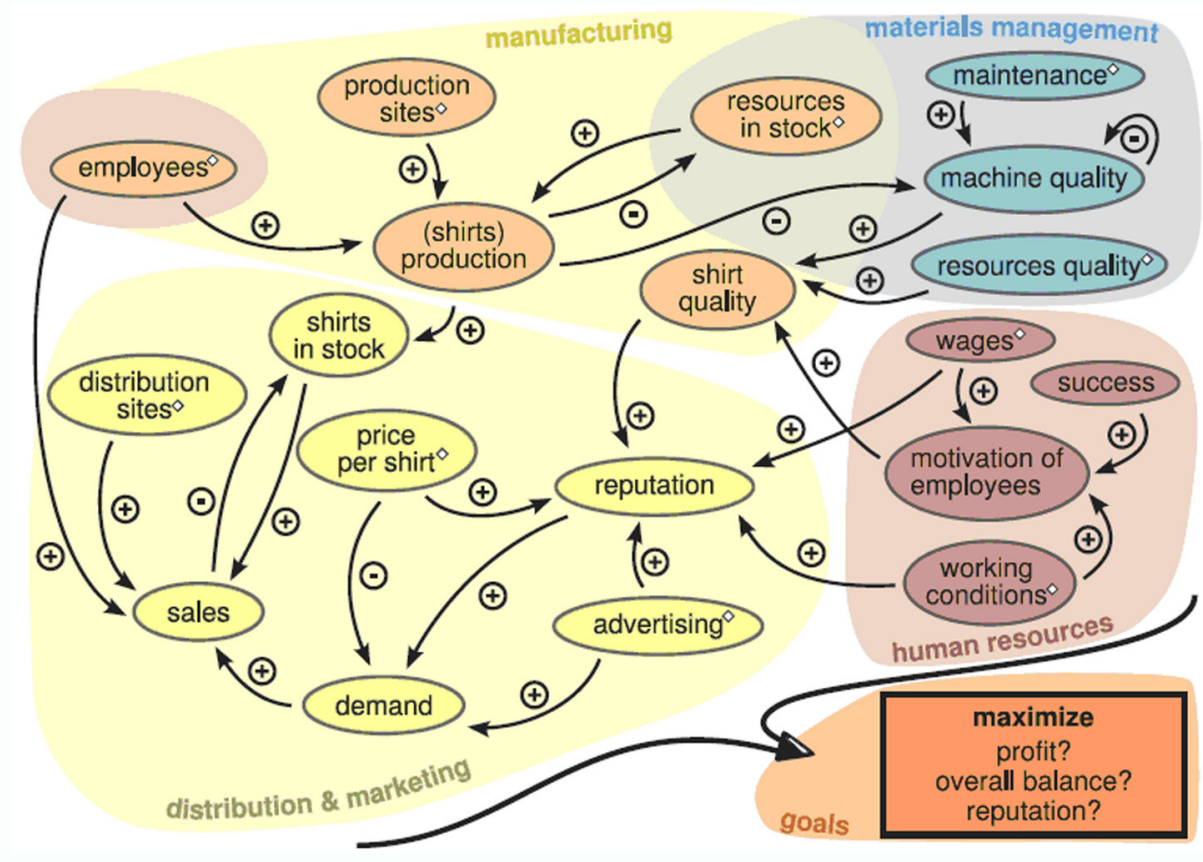

Figure 1. The structure of the CPS scenario TAILORSHOP, with the positive and negative dependencies between the influential variables. Diamonds represent the participant's control possibilities. (Engelhart, Funke \& Sager, 2011)

- volume 4, no. 1 (Winter 2012) 
1. The complexity of a system ${ }^{2}$ may be defined as the number of elements and relations of the system (see Funke, 1985). As Dörner (1989) stated, "the complexity of a domain of reality is the higher, the more features there are and the more these features are interdependent" (Dörner, 1989, p. 60, translated by the authors).

2. A problem is considered to exist, "when a living creature has a goal but does not know how this goal is to be reached. Whenever one cannot go from the given situation to the desired situation simply by action, then there has to be recourse to thinking" (Duncker, 1945, p.1). Dörner has gone into more detail when he emphasized that "barriers" between the given situation and the desired goal state, i.e., the lack of knowledge, can be further classified according to the amount of (a) ignorance of the means/operators applicable, and (b) lack of concreteness concerning the goal state (see Dörner, 1976, or Funke, 2003).

3. Problem solving can be defined as successfully searching for an operation or a series of operations in order to transfer the given actual state of the system to a goal state (Newell \& Simon, 1972; Dunbar, 1998).

Based on these three concepts, CPS can be defined as a kind of problem solving, with the problem itself (the structure of (a) the external problem representation and/or (b) the mental representation of the problem), or the process of its solution having to be formalized as a set of many highly interrelated elements, i.e., a complex system. According to Halford, Wilson and Phillips (1998) the complexity of relations can be quantified by the number of variables related to each other: For example, the mental representation of a criterion $y$ depending on a predictor $x$ could be expressed as a binary relation $r(y, x)$, whereas a dependency on multiple predictors could be represented as a relation of higher rank, e.g., the ternary relation $r(y, x 1, x 2)$, and thus would be considered more complex. Structures more complex than quaterny relations are assumed to have to be processed by either conceptual chunking or segmentation in order to not exceed human processing capacity (Halford et al., 1998).

One famous example for CPS - that can be considered complex because the structure of the external problem representation (see Figure1) is to be formalized as a complex system-is the TAILORSHOP (see, e.g., Funke, 2003), a computer simulated scenario of a small organization involved in shirt production. Originally programmed by Dörner in the 1980s on his calculator it was implemented on many platforms and used in a variety of contexts.

In this scenario, the problem solver takes the role of managing a small tailorshop, deciding what actions to take or what information to gather, aiming at the maximization of the capital at the end of each simulated month (a global goal which is dependent on a set of conflicting subgoals). On an abstract level, the structure of the TAILORSHOP scenario is formalized as a complex dynamic ${ }^{3}$ system, consisting of many highly interrelated vari-

$2^{2 \prime} \mathrm{A}$ system is understood as an entity functionally closed and separated from its environment, consisting of elements which are in interaction with each other. Systems can be open to processes of exchange with their environment. Depending on the depth of system analysis there can be different hierarchical layers discriminated within systems and heterarchical interactions between systems." (Strunk \& Schiepek, 2006, p.102) 
ables (see Funke, 2003). In the literature on CPS, it is mostly the structure of the external problem representation that is considered complex. So a problem usually is considered being of a certain complexity, even if it might seem less complex to problem solvers with more expertise (as well as it is considered being of a certain difficulty, independent of the ability of a problem solver). This view is essential in order to understand the research on some of the most noteworthy aspects of CPS: For instance, using parsimonious but viable heuristics (see research on decision making strategies) and representations (see research on information reduction) are often considered most important for coping with complex problems (see Gigerenzer \& Brighton, 2009; Gonzalez \& Lebiere, 2005; Klauer, 1993). To efficiently cope with complex problems using adequate heuristics and representations (see research on intelligence) the problem solver has to either use or acquire sufficient implicit or explicit knowledge about the problem (see research on expertise). Especially when the problem is not presented as a set of nameless and abstract variables, but embedded in a plausible semantic context (like the TAILORSHOP, described above), prior knowledge about the elements to focus on or about the strategies to apply best, helps in reducing the problem space that has to be searched through for a solution to the problem (see research on human problem solving).

On the following pages we will review what is known about these most important aspects of CPS, and how it fits together in an integrative process theory of CPS. Therefore we will review and summarize findings of five fields of research that have contributed most to the understanding of CPS: (a) human problem solving, (b) expertise, (c) decision making strategies, (d) information reduction and (e) intelligence.

\section{Human Problem Solving}

The most general conception of problem solving up to now, which might as well be expanded and applied to CPS, has been Newell's and Simon's (1972) Theory of Human Problem Solving. The theory was proposed to explain findings on simple static and welldefined problems not as complex as the TAILORSHOP, but Newell and Simon already addressed all the aspects necessary to solve problems of arbitrary complexity.

Following the authors, some of the most important aspects of human problem solving may be summarized as follows:

1. Human problem solving starts with constructing an internal representation of the external problem statement, a "problem space" (i.e., a set of possible states of the problem, given the initial state, the applicable operators, and certain goal states). Which operators can be considered applicable might be different for problem solvers of different expertise and intelligence (see Newell \& Simon,

\footnotetext{
${ }^{3} \mathrm{~A}$ dynamic system is a system, that contains a vector of variables, that is dependent on former states of the same vector, e.g., $\mathbf{Y}(\mathrm{t})=f(\mathbf{Y}(\mathrm{t}-1))$ (see Funke, 1985, p.4)
} 
1972).

2. Given an internal representation of the problem, a method for reaching the current goal is being searched for. General searching algorithms (like "hill-climbing", or "means-end-analysis") are distinguished from more domain specific methods (like "take the hammer to get the nail into the wall")

3. Using a method can change the external problem as well as the internal representation. Of course, changes in the environment or the consequences of a method may lead to new (sub-)problems or new possible solutions. Methods also can be aborted when metacognitive processes do interfere. When a method does not lead to a goal state, (1) another method can be tried, (2) the internal representation may be changed, i.e., the problem may be reformulated, or (3) the attempt of solving the problem may be aborted.

When it comes to CPS constructing a parsimonious but viable internal representation is far from trivial (in contrast to the problems Newell and Simon used in their studies, where a correct internal representation is usually assumed to be given). Usually a problem solver has to actively acquire knowledge about the complex problem by systematically interacting with it (see Funke, 2001) as the initial assumptions about the structure of the problem are mostly false or incomplete (Dörner, 1989). Often the problem solver has to define one or more of the problem's components him- or herself based on aspects like prior knowledge (e.g., experience with analogous problems, or generalized schemas for this kind of problems) and features of the task (Novick \& Bassok, 2005) and usually building a viable internal representation of a complex problem involves processes like rule induction (Simon \& Lea, 1974), generating and testing hypotheses (Klahr \& Dunbar, 1988) and causal learning (Buehner \& Cheng, 2005).

Fortunately, there are some theories that elaborated on certain aspects of knowledge acquisition in more detail (e.g., explaining when active information generation takes place and how it leads to better representations): Ohlsson (1992) proposed a Theory of Representational Change. When the current problem representation does not cue the operators sufficient to solve the problem, Ohlsson speaks of an "impasse". An impasse can be broken, when the problem representation is changed, as a different problem representation might cue other concepts in long-term memory. Representational change may occur in different ways: (1) Elaboration of (or search for additional) information about the problem; (2) constraint relaxation, i.e., removing inhibitions on what is regarded as permissible; (3) re-encoding, i.e., reinterpreting the problem representation. With his theory of representational change he emphasized the importance of a viable problem representation for solving problems and thus elaborated on an aspect of special importance to CPS. MacGregor, Ormerod, and Chronicle (2001) proposed that changes in the problem representation and in strategy use may occur due to monitoring processes, when the rate 
of progress is perceived to be too slow to solve the problem in time. According to their theory, it is not as much the impasse, but the perception of an impasse (or even an unacceptable slow-down in progress) that leads to phenomena like restructuring, considering new operators and insight.

Simon and Lea (1974) have further elaborated on Newell's and Simon's (1972) concept of problem space in a way that also proved to be fruitful for the CPS research. They conceptualized the problem space as divided into a rule-space (containing possible rules of the problem) and an instance-space (containing possible states of the problem) with information in each space guiding the search in the other space (see also Klahr \& Dunbar, 1988 , for an extension and application of the dual-search concept to the complex field of scientific discovery). This conception sheds light on how instances and rules of the problem are explored (i.e., how a solid representation of a complex problem is built) and can be considered fundamental in modeling the influence of knowledge about instances and structural knowledge on problem solving as it is considered by research on the influence of expertise on CPS.

In summary, information processing theories on human problem solving have proposed some useful ideas and assumptions that are most relevant when building a process theory of CPS. E.g., they try to explain when information generation and elaboration takes place, how it leads to viable internal representations (or models) of the problem system, and how the internal representation of the problem determines the solution strategies applicable. Especially the distinction of structural knowledge and knowledge about instances proved to be very fruitful for thinking about the influence of expertise on CPS. The next section will further elaborate on this distinction, and propose the most influential theories on how different kinds of knowledge may influence the process of CPS.

\section{Expertise}

There is a large quantity of research on differences between experts and novices of a certain knowledge domain concerning the influence of different kinds of domain-specific knowledge on CPS. In fields as different as reading, writing, arithmetic, mechanics, policies, jurisdiction, management, or debugging (for an overview see Sternberg \& Frensch, 1991) there has been a lot of research on the processes and kinds of knowledge involved in CPS. What could have seemed to be a turning away from general aspects of problem solving in favor of more domain-specific problem solving strategies nonetheless produced a deep insight in some general effects of expertise on general problem solving.

For instance:

- Experts can (a) apprehend a greater number of elements in working memory and (b) retain these elements for a longer duration, when the elements are part of a meaningful configuration within their domain of expertise (see expertise 
wide-span memory; Horn \& Blankson, 2005).

- Experts classify problems according to deep features, relevant to the solution, rather than superficial features (Chi, Feltovich, \& Glaser, 1981);

- There are differences in the semantic memory of experts, compared to novices regarding, e.g., the associations between concepts (Chi et al., 1981);

- Experts are faster in solving problems if they are asked to do so (Chi, Glaser, \& Rees, 1982);

- Experts are more precise if not working under uncertainty (Johnson, 1988);

- Experts seem to have better metacognitive abilities (like self-monitoring) (Larkin, 1983).

Consequently the process of gaining expertise concerning a certain problem via building explicit and implicit representations of the problem at hand was of special interest to the research community and stimulated a lot of interesting theoretical and empirical results on CPS as will be outlined in the next paragraphs.

One of the most influential theories on (1) gaining explicit declarative knowledge and on (2) the effects of expertise on problem solving and learning is John Sweller's Cognitive Load Theory (CLT). Sweller (2005) assumed that the human cognitive architecture, in order to efficiently adapt to dynamic environments, consists of (a) a working memory, which is capable of processing (e.g., combining, contrasting or manipulating) two to four elements/chunks simultaneously (see also Halford et al., 1998), and (b) a long-term memory with almost unlimited capacity for chunks of declarative knowledge. To spare working memory capacity for processes relevant to learning (i.e., elaboration and selfexplanation) work load has to be as small as possible. In CLT there are three kinds of work load differentiated: (a) intrinsic load, resulting from the complexity of the task (dependent on learner's expertise and the interactivity of elements to be processed); (b) extraneous load, determined by demands resulting from suboptimal instructional design; (c) germane load, resulting from effortful learning and elaboration and leading to schema construction or automation (Sweller, 2005). Schemata are assumed to be stable representations of transient experiences, assumed to (1) guide future recognition (assimilation) of similar experiences, (2) initiate appropriate actions and expectations and/or (3) be accommodated to new experiences if necessary (see von Glasersfeld, 1997). When it comes to problem solving, according to Sweller (2005), an expert can assimilate what seems to be multiple elements to a novice under one single schema to spare work load via working on a chunk of higher order (i.e., a chunk containing chunks) instead of having to work on multiple chunks. Furthermore, schemata are assumed to have an executive function, guiding the problem solving process of experts, whereas novices have to rely on inefficient and more general search strategies causing additional work load. Therefore, gaining expertise about the structure and the dynamics of the problem plays one of the most important roles in 
solving complex problems, as expertise (a) helps to reduce intrinsic load given a certain interactivity between the elements of the task and (b) is assumed to moderate the usefulness of certain strategies and the effect of problem characteristics (this moderating effect is commonly referred to as the expertise reversal effect, see Kalyuga, 2007). The principles derived from CLT have successfully been applied to CPS tasks such as air traffic control and interactive games (for an overview see Osman, 2010).

Whereas CLT has its focus predominantly on explicit declarative chunks of knowledge, other approaches have emphasized the importance of implicit knowledge for CPS. The importance of implicit knowledge in controlling dynamic systems was made clear by Berry and Broadbent (1984) who found that practice and learning did enhance performance, although it did not lead to verbalizable knowledge about the system structure. Broadbent and colleagues examined implicit learning in system control using minimal complex dynamic systems like the SUGAR FACTORY (which is based on the equation Pt $=2 * \mathrm{~W}-\mathrm{Pt}-1+\mathrm{e}$; where $\mathrm{W}$ is the number of workers, $\mathrm{P}$ is the amount of sugar produced at a moment in time $t$, and $\mathrm{e}$ is a random error term. (See Berry \& Broadbent, 1984). They proposed an instance-based theory of system control, claiming that successful interactions with a dynamic system were stored in memory as a kind of "look-up table" of instances, containing information about (a) the perceived state of the system and (b) the input necessary to reach the target level (Broadbent, Fitzgerald, \& Broadbent, 1986). Decisions about what action to execute in a given situation can then be based on the instance matching the perceptual properties of the current situation best. A lot of instance-based theories have been proposed since then (e.g. Dienes \& Fahey, 1995; Lebiere, Wallach, \& Taatgen, 1998; Logan, 1988), each able to reproduce the behavior of participants trying to solve the SUGAR FACTORY. A modern version of the instance-based learning theory within the framework of the cognitive architecture ACT-R was proposed by Gonzalez, Lerch, and Lebiere (2003) in order to explain decision making in complex, dynamic situations especially under uncertainty (Gonzalez \& Lebiere, 2005). Gonzalez et al. (2003) assumed that every decision is stored as an instance, i.e., as a chunk of knowledge with slots containing (a) a set of features of the situation, (b) the decision made, and (c) the expected utility of this decision. In the absence of instances similar to the current situation, the decision maker is assumed to rely on simple heuristics for making his or her decision (e.g., random choice). When instances similar to the current situation are retrieved from memory, the decision maker is assumed to rely on the alternative with the highest aggregated utility after she or he has evaluated a certain amount of alternatives (depending on factors like the aspiration level of the decision maker and the perceived urgency of decision, in regard to the time remaining). After a decision was made, the utility-slot of the decision is updated according to the outcome of the decision via a feedback process.

Even though instance-based learning often leads to successful system control (e.g., in systems like the SUGAR FACTORY), it is of limited transferability as it does not involve infor- 
mation about the properties of the system structure (Berry \& Dienes, 1993). See Figure 2 for the difference between knowledge about (a) an instance of a system and (b) the structure of the system. Structural knowledge is transferable and allows for building expectations about the consequences of certain decisions and actions in a given situation. It may be action-guiding even in hypothetical situations or in situations never encountered before.

As Schoppek (2002) pointed out, the usefulness of instance knowledge also depends on the size of the problem space, i.e., on the number of possible input- and output-states of a system as well as their relations. He emphasized that additional knowledge about the system structure becomes necessary when larger systems have to be controlled as an

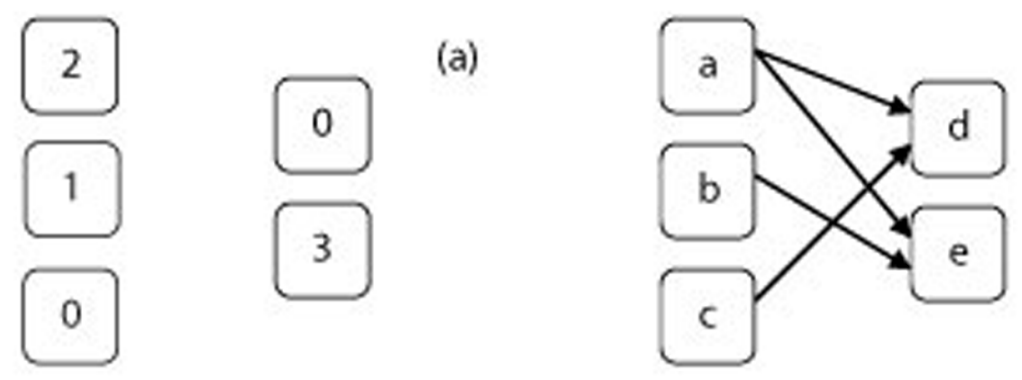

(b)

Figure 2. Visualization of two different kinds of knowledge about an exemplary system containing 5 variables: (a) An instance of the system (consisting of a set of numerical values) and (b) the structure (consisting of the relations between abstract variables).

instance-based model would require a tremendous amount of input-output-instances to cover a substantial part of the problem state when the system that has to be controlled consists of many input- and output-variables. Structural knowledge may be of use even in situations never seen before. Empirically this assumption proved to be valid: Funke (1993) studied slightly more complex systems (with three input- and three output-variables) and indeed found significant correlations between structural knowledge and control performance (as well as effects of the system's complexity on both measures). Quesada et al. (2005) supplemented Schoppek's view as they mentioned the moderating role of expertise: Experts may be able to have sufficient implicit instance knowledge even about large systems.

The acquisition of structural knowledge about complex systems seems to depend on conscious thought and mental effort (corresponding to the germane load; Sweller, 1988). The acquisition of structural knowledge thus may be fostered by the intention and the opportunity to explore the system before or instead of having to achieve a certain goal (Sweller, 1988; Vollmeyer, Burns, \& Holyoak, 1996). Instance knowledge, on the other hand, seems to be acquired without germane load, automatically as a result of practice (Schop- 
pek, 2002). Logan (1988) pointed out that in the absence of implicit knowledge finding the solution to a problem (i.e., the response to a stimulus) requires conscious thought and the application of rules. Only after a vast amount of practice the correct response can be retrieved rapidly and automatically. So in the absence of relevant implicit knowledge there have to be general heuristics and explicit knowledge guiding the course of problem solving (see Gonzalez et al., 2003; Sweller, 2005).

Each interaction with the system may be considered generating an instance that could be stored in memory to implicitly guide future decisions in the face of similar system states-under certain circumstances (factors like time pressure, stress, uncertainty, and high cognitive load may foster the reliance on instance knowledge. See Gonzalez and Lebiere, 2005). In addition to knowledge about instances, systematic strategy use may allow inference of knowledge about the system structure (see section on decision making strategies) which might come in handy under different circumstances (e.g., when trying to reach system states never seen before, maybe due to a large problem space and insufficient expertise).

So after having considered different kinds of knowledge that can be assumed to have an influence on CPS, in order to build a process model of CPS it seems promising to further examine (a) the circumstances determining which kind of knowledge (e.g., structural or instance based) problem solvers usually rely on to make their forecasts, plans and decisions, and (b) what strategy is chosen when no knowledge about the correct solution to a problem is available yet. Answers to this question were proposed in the field of research on decision making and will be reported in the next section.

\section{Decision making strategies}

Research on decision making has developed a set of decision making strategies containing viable strategies and heuristics for (a) generating relevant information and (b) making good forecasts and decisions in complex environments. When the goal is to specify an input or a series of inputs in order to regulate certain output-variables of a complex system, each possible input vector (e.g., an action in a complex scenario) can be considered an option, with several expected consequences (e.g., changes in the output variables). Each consequence may have a subjective utility and an expected probability specific to the current context (i.e., the consequences of an action may be of different perceived use and certainty, dependent on factors like the perceived features of the situation). In complex scenarios there seldom can be an exhaustive evaluation of all possible options and their weighted consequences (due to time pressure and the tremendous amount of variables that would have to be considered). Instead, decisions have to be based on strategies using less information and only a small amount of computation (e.g., by taking the option

which has the highest value on the most important consequence - the so-called "take the

- volume 4, no. 1 (Winter 2012) 
best"-heuristic). With regard to CPS, it is of special interest to note that simple heuristics like "take the best" or simple tallying can actually achieve higher accuracy in predicting the best outcome than more complex algorithms under certain circumstances - e.g., low predictability of a criterion, combined with small sample sizes relative to the number of available cues, and dependency between cues (Gigerenzer \& Brighton, 2009). So when it comes to predicting new observations (instead of just fitting data already observed) sometimes the "less-is-more"-approach holds to be true and it proves to be more accurate to make decisions based on only one good reason (i.e., "take the best") than using tallying, multiple regression or even heavy-weight nonlinear strategies like neural networks (Gigerenzer \& Brighton, 2009). Therefore, the question is not as much which strategy is the best but which is the best in a certain environment, i.e., under certain conditions.

The applicability and/or the usefulness of some strategies-their ecological rationality (Gigerenzer \& Brighton, 2009)—can depend on the existence of prior experiences with the system, on the amount of detailed structural knowledge about the values and weights, on knowledge about the alternatives available, etc. Thus, memory on the one hand constrains the set of heuristics applicable (each long-term and working memory can be considered to constrain what is possible in a certain situation) and on the other hand "selects" heuristics that are likely to yield accurate decisions in a mostly unconscious process (Gigerenzer \& Brighton, 2009). Furthermore, the ecological rationality of a heuristic in a given environment is assumed to depend on factors like the structure of the environment and feedback, amongst others. According to Rieskamp and Otto (2006), the ecological rationality can be learned by the decision maker via simple reinforcement learning. When goal-oriented decisions are dependent on former decisions and their consequences in an environment that may change both spontaneously or as a consequence of earlier actions, it is commonly referred to as Dynamic Decision Making (DDM; Edwards, 1962). Busemeyer (1999) has given an overview of the research on DDM, stating that on the one hand human performance usually can be considered suboptimal, but that on the other hand systematic learning effects were found in almost all of the studies reviewed.

So, during the process of CPS, problem solvers seem to increasingly rely on strategies that are efficient and ecologically rational, i.e., they (1) rely on the correct solution if it is known automatically (instance knowledge), elsewise (2) search for a solution based on the current problem representation (structural knowledge), or (3) gather new information about the problem (e.g., via random or systematic interaction with the system, via asking an expert, etc.). This conception seems to be consistent with the "Elshout-Raaheim-Hypothesis" (Leutner, 2002), stating that correlations between problem solving and intelligence may be dependent on knowledge about the system in an inverted-U-shaped way (i.e., the correlation may be minimal when prior knowledge is very high or very low as consequently no reasoning is necessary in these cases). In some cases it might even be an option to abandon certain goals due to their unattainability (see Brandtstädter, 2007), or to give up 
the attempt of a rational solution.

When it comes to gathering information (e.g., when the structural knowledge about the problem proves to be insufficient), some strategies may be especially useful for generating viable structural knowledge about the system. As Vollmeyer et al. (1996) pointed out, systematicity in strategy use allows a problem solver to coherently infer the consequences of single interactions, i.e., to build viable structural knowledge about parts of the system structure. For example, following Tschirgi (1980), to "vary one thing at a time" (while setting the other variables on a constant value like zero)_commonly referred to as the VOTAT-strategy-may be a strategy useful to systematically identify the effects of independent (exogenous) variables on dependent (endogenous) variables in certain scenarios (especially when each exogenous variable was contrasted to the other ones at least one time. Setting the increments of all input variables to a value of zero from time to time may facilitate the detection of eigendynamics and indirect effects). Systematic strategy use and generating (as well as using) structural knowledge might be especially important in complex systems when there is no (or even cannot be) sufficient implicit knowledge about a correct solution of the problem. But as human cognitive resources are limited, even detailed and extensive structural knowledge about all the aspects of a complex system may not be fostering CPS per se as they may overcharge the human working memory. Based on this crucial aspect of complex problems the following section proposes the most influential theories on how and why information reduction is an essential aspect of CPS.

\section{Information reduction}

As large amounts of knowledge may overcharge human processing capabilities, a most important aspect of coping with complexity is information reduction. Klauer (1993) proposed a theory of information reduction in CPS. Based on the assumption that problem solving involves processes of using certain searching strategies (implicit procedural knowledge) applied to a mental representation of the problem (explicit declarative knowledge) demanding resources of working memory with its limited capacities, Klauer stated, based on his empirical findings, that it was mainly the (declarative) representation of the problem that was reduced in case of capacity overload. Two mechanisms for reducing processing load imposed by complex representations are conceptual chunking and segmentation (Halford, et al., 1998). The consequence is a parsimonious representation.

As Gonzalez and Lebiere (2005) pointed out, the development of effectiveness in DDM involves an increasing selectivity in the use of information, via focusing on relevant features whereas ignoring irrelevant features of the situation. The relevance of features may be determined based on explicit or implicit knowledge. For instance, in their IBLT (see section on expertise) the relevant features of a current situation are assumed to stand 
out in the recognition process, because they resemble cues in the instances stored in memory. With increasing practice the common features of past instances, similar to the current situation, can be abstracted to guide the attention to the important aspects of the situation (Gonzalez \& Lebiere, 2005). This is consistent with the predictions of the chunking/template theories (Chase \& Simon, 1973; Simon \& Gobet, 1996) and the information reduction hypothesis (Haider \& Frensch, 1996).

Newell and Simon (1972) considered a method for planning that emphasized the importance of information reduction regarding irrelevant differences and operators in order to approximately find a way through huge problem spaces. This method consisted of (1) abstracting from details of objects or operators, (2) generating an abstract problem space, (3) searching for a solution in the abstract problem space, and finally (4) trying to map the abstract solution on the concrete problem space with all its details. These considerations are of special importance to CPS, where the abstraction from irrelevant details often is the only way to make adequate forecasts of the system's behavior in spite of the tremendous amount of variables and relations involved. Gaschler $(2009$, p.5) stated, that "research on information reduction emphasizes practice-related changes of which rather than how information is being processed. Information reduction applies in situations in which tasks contain both relevant and irrelevant information, and denotes a change from a strategy that is based on exhaustive processing of all elements of a task to a strategy that skips the irrelevant task components".

To summarize research on information reduction, in CPS omitting irrelevant task components and finding a parsimonious representation of the problem may enable and foster the search for a solution to a complex problem. Because the search for a solution based on a viable parsimonious model of the problem involves processes like inductive and deductive reasoning, that are commonly subsumed under the concept "intelligence", the next section of this article will review the empirical and theoretical findings on how different aspects of intelligence influence CPS before we will integrate the findings reported so far in a process model of CPS.

\section{Intelligence}

Theoretically, general intelligence may be defined as "the global capacity of a person to act purposefully, to think rationally, and to deal effectively with his environment" (Wechsler, 1944). Originally, general intelligence as a concept was proposed to explain covariance between a wide range of cognitive tasks, and reasoning as well as problem solving have traditionally been a part of the definition (Sternberg, 1982). Research on intelligence is about the cognitive processes involved in solving tasks and problems and thus may contribute to a profound understanding of CPS. According to the very broad conceptualization of intelligence, it seems quite natural to ask about (1) the amount of variance in CPS 
performance that can be explained by traditional tests of general intelligence, and about (2) the facets of intelligence that may be most relevant for CPS.

At the beginning of CPS research intelligence surprisingly seemed to be only loosely correlated with performance in complex scenarios (see e.g., Wenke et al., 2005). From today's point of view this lack of evidence in the early days of research on CPS can partially be attributed to the insufficient psychometric qualities of early measures of performance in complex scenarios (e.g., in the TAILORSHOP neither the absolute capital values at the end of each simulated month, nor the changes of capital, but the sum of changes proved to be a reliable and valid measure for CPS performance; for a review, see Danner et al., 2011). According to Danner et al. (2011), using a reliable performance measure revealed substantial correlations of performance in the TAILORSHOP with intelligence measured by Advanced Progressive Matrices ( $r=.31, p=.001)$, job performance rated by supervisors $(r=.19, p=.025)$, and other measures for CPS performance $(r=.31, p<.001)$. Süß, Oberauer, and Kersting (1993) also found significant correlations of a TAILORSHOP performance measure with the intelligence facet capacity measured by the BIS test (Jäger, Süß, \& Beauducel, 1997). In another influential study, Wittman and Süß (1999) also revealed a substantial effect of working memory capacity on the performance in different CPS scenarios, and the authors also stated, that correlations between the different scenarios became about zero when system-specific knowledge and intelligence were partialed out. According to these findings, working memory capacity and the processes involved in generating systemspecific knowledge seem to be the most important facets of intelligence in explaining CPS performance. There is currently an ongoing debate if the generation and application of knowledge in CPS address some facets of general intelligence that are not yet addressed for by traditional intelligence tests (see Wenke et al., 2005).

Generally, traditional intelligence tests, aiming primarily at speed and quality of human symbol processing (i.e., fluid reasoning) as well as working memory capacity, were criticized for their primary focus on the results instead of the process of efficient problem solving behavior (Dörner, 1986). Additionally Horn and Blankson (2005) criticized that there may be more complex "expertise abilities" (Horn \& Blankson, 2005, p. 60) different from fluid reasoning, working memory and cognitive speed, which are not adequately addressed for by the tests that are assumed to indicate human intelligence. Putz-Osterloh (1981) stated that the most important differences between the demands of classical tests for measuring intelligence and complex problems were the (1) polytelic situation, the need for an (2) active search for relevant information, for (3) specifying concrete goal states and for (4) choosing productive actions, as well as for (5) a greater relevance of prior knowledge in the latter case. According to this line of argumentation, there are facets of general intelligence that are not yet accounted for by traditional intelligence tests.

With his concept of operative intelligence Dörner (1986) emphasized the importance of examining not only speed and precision of some of the basic intellectual processes, but 
also the more formative aspects of problem solving, for example (1) circumspection (e.g., anticipation of future and side effects of interventions), (2) the ability to organize cognitive operations (e.g., knowing when to do trial-and-error and when to systematically analyze the situation at hand; when to use exhaustive algorithms and when to rely on heuristics, when to incubate an idea etc.) or (3) the availability of heurisms (e.g., being able to build helpful subgoals, to constrain the problem space efficiently). This list of examples is not exhaustive, but it gives an idea of what is meant by the "operative" aspects that are not adequately addressed by traditional intelligence tests but may still be considered relevant for an organized course of intellectual processes (Dörner, 1986). With its explicit focus on gaining and using information and knowledge about the cognitive operations adequate, operative intelligence can be considered one of the most relevant expansions of intelligence as it is measured with current measurement devices:

Intelligence in a problem solving situation turns out to be being able to collect information, to integrate and structure information goal-oriented, to make prognoses, to plan and to make decisions, to set goals and to change them. To achieve all this, an individual has to be able to produce an organized series of information processing steps, flexibly adapting these steps to the demands of the situation, and then it is intelligent. (Dörner, 1986, p. 292; translated and emphasized by the authors).

The facets of operative intelligence emphasized in the characterization just given closely resemble the facets most relevant for coping with the characteristic features of complex problems (see Burmeister, 2009; Dörner, Kreuzig, Reither, \& Stäudel, 1983; Funke, 1992, 2001, 2003, 2011):

1. the complexity of the structure (calling for information reduction),

2. the interconnectedness of the variables (calling for building a model of the most relevant effects),

3. the polytely of the task (calling for evaluation and for setting priorities),

4. the intransparency of the situation (calling for systematically generating information), and

5. the dynamics of the system (calling for Dynamic Decision Making).

These characteristic features of complex problems and the corresponding facets of CPS (see Funke, 2001) can be considered a fruitful starting point for measuring operative intelligence, which in turn might be the most important determining factor of CPS performance. According to Dörner (1986) the most relevant facets of operative intelligence could be measured evaluating and quantifying the "questions" (meaning behavior to explore the 
system actively generating information) and "decisions" (meaning behavior to control the system goal-oriented) of testees solving complex problems (see the MicroDYN approach presented in Greiff, in press, or Wüstenberg, Greiff, \& Funke, in press, for a promising attempt to do so). As Dörner (1986) emphasized, the demands of CPS situations are characteristically intertwined in an inseparable way, and the problem solving process has to be studied as a whole because the parts are interacting with each other and hardly can nor should be examined in isolation:

1. Information retrieval and information integration: The problem solver needs a model adequately representing the system and the goal state to aim at. Therefore she or he has to systematically generate, gather, and integrate information to adjust this model to the system.

2. Goal elaboration and goal balancing: The problem solver has to specify and substantiate the often vague and global goals she or he wants to achieve. If some specified goals turn out to be contradictory, she or he has to find a satisfying trade-off or balance in only partially reaching the goals.

3. Action planning and decision making: The problem solver has to decide what actions to execute, i.e., what decision making strategies to apply (see section on decision making strategies), and which kind of knowledge to rely on (see section on expertise). By forecasting future developments given the system's prior states and her or his own actions she or he can efficiently plan her or his next steps (e.g., chains of consecutive actions with each action building on the results of the previous one).

4. Self management: The problem solver may have to face time pressure, stress, and frustration as well as conflicts between his inner values. She or he has to manage these non-cognitive affordances by either changing the system or his own behaviors and habits.

So after these considerations about how efficient CPS may look like and what facets of intelligence may influence the CPS performance we want to proceed by integrating the contributions of all the fields of research mentioned above in a process theory of CPS.

\section{Discussion}

After reviewing some of the most important fields of research on CPS, and based on the definition given above, we are now going to summarize the interdisciplinary findings in a process theory of CPS, concluding with a short outlook for upcoming research. CPS can be understood as the process of solving problems that have to be considered "complex" (i.e., containing many highly interrelated elements). For instance, every scientist, who wants to 
describe, explain, and predict a complex system by means of her or his hypotheses (containing a parsimonious but viable subset of all variables possibly relevant) might be facing a complex problem. A mayor of a city as well as a manager of an organization or a policy maker trying to get rid of climate change, each may be considered as having a complex problem to cope with. Trying to make a modern computer do what it is supposed to can turn out to be a complex problem as well as changing certain settings of an unknown mobile phone device. The process of CPS usually consists of different phases: (1) knowledge acquisition and (2) goal-oriented knowledge application (Leutner, Wirth, Klieme, \& Funke, 2005). Usually a problem solver switches between these phases in a complex way:

1. At first, the problem solver has to acquire knowledge about the problem.

a. The problem solver is assumed to explore the system's behavior using a strategy that (a) she or he knows of and (b) seems to be most ecologically rational to her or him (e.g., random or systematic interaction with the system, reading the instructions, asking an expert, etc.).

b. The exploration leads to (a) knowledge about the system's states and the actions taken (instance knowledge) as well as (b) an internal representation of the problem, containing the most important elements and relations of the system (structural knowledge) which usually is inferred from the instance knowledge.

c. As the capacity of the problem solver's working memory is limited, the internal representation is object to information reduction. Relations and elements that prove to be less relevant for system control in the course of exploration are assumed to be omitted in order to allow more efficient planning and forecasting.

2. When the problem solver has a certain amount of knowledge about the problem that has to be solved, she or he is assumed to apply the knowledge in order to reach her or his goals.

a. The problem solver is assumed to use her or his internal representation to make forecasts about the system's dynamics in order to decide (a) if she or he has to intervene and (b) what intervention will have acceptable consequences in the current situation. When the current situation cues the correct intervention immediately (due to instance knowledge), the problem solver is assumed to rely on her or his instance knowledge instead.

b. Monitoring processes are assumed to detect (a) the progress in solving the problem and (b) the implications of feedback from the environment for the problem representation. When the problem representation proves to be not viable for reaching the goals in time, the problem solver is assumed to either switch back to knowledge acquisition or to change the 
goals (depending on factors like the importance of the goals and on the assumed effort of further knowledge acquisition).

This process theory of CPS summarizes what is known about the most important aspects of CPS and is based on the theoretical and empirical contributions of the interdisciplinary field presented in the previous sections. As CPS is a rather abstract concept, further research is needed to specify the process of CPS concerning concrete operationalizations of complex problems (e.g., handling a complex mobile phone may be represented in other ways than regulating an economic system or managing a tailorshop). Concerning this, it seems to be a fruitful approach to build cognitive models of the CPS process (e.g., Schoppek, 2002) in order to develop a deeper understanding of CPS processes taking place in real life.

But even on a more abstract level our theory on the CPS process may be subject to further research. It may be seen as a starting point for further experiments, in order to gradually improve our understanding of what CPS is and how it works (e.g., experimental psychology may further contribute knowledge about variables or interactions with a significant impact on the process of CPS). Psychometrics may contribute to a better understanding of CPS by developing reliable and valid measures for the processes that are assumed to be important for efficient and intelligent CPS (Greiff, in press; Wüstenberg, Greiff, \& Funke, in press). Those measurement devices in turn can be used to test process theories on CPS in more detail.

Our conception of CPS is inspired by the pioneering works of Dörner, especially by the concept of operative intelligence (Dörner, 1986) and the considerations of Funke (2001), emphasizing (a) information generation (due to the initial intransparency of the situation), (b) information reduction (due to the overcharging complexity of the problem's structure), (c) model building (due to the interconnectedness of the variables), (d) dynamic decision making (due to the dynamics of the system), and (e) evaluation (due to many, interfering and/or ill-defined goals). In unison with Dörner we want to emphasize that in order to develop a sufficient understanding of the problems humans have to face in their everyday lives, research on problem solving has to further elaborate on complex problems, with both a large amount of possible actions for the problem solver, and a lot of uncertain and surprising consequences in naturalistic environments. The more we learn about the process of problem solving, the more we have to acknowledge the complexity of both the process and the kind of problems that are involved in realistic problem solving in naturalistic environments.

\section{References}

Berry, D. \& Dienes, Z. (1993). Towards a working characterization of implicit learning. In 
D. Berry \& Z. Dienes (eds.), Implicit learning: Theoretical and empirical issues. Hillsdale, NJ: Erlbaum.

Brandtstädter, J. (2007). Das flexible Selbst. Selbstentwicklung zwischen Zielbindung und Ablösung [The flexible self. Self development between attachment and detachment]. München: Elsevier.

Berry, D.C., \& Broadbent, D.E. (1984). On the relationship between task performance and associated verbalizable knowledge. Quarterly Journal of Experimental Psychology, 36A, 209-231.

Broadbent, D., Fitzgerald, P., \& Broadbent, M. H. P. (1986). Implicit and explicit knowledge in the control of complex systems. British Journal of Psychology, 77, 33-50.

Buehner, M.J. \& Cheng, P.W.(2005). Causal Learning. In Holyoak, K.J. \& Morrison, R.G. (Eds.), The Cambridge Handbook of Thinking and Reasoning (pp. 143-168). Cambridge, UK: University Press.

Burmeister, K. (2009). Komplexes Problemlösen im Kontext angewandter Eignungsdiagnostik [Complex Problem Solving in the context of applied diagnostics of suitability]. Universität Greifswald, Philosophische Fakultät.

Busemeyer, J.R. (1999). International Encyclopedia of the Social and Behavioral Sciences: Methodology. Mathematics and Computer Science. Amsterdam: Pergamon.

Chase, W. G. \& Simon, H. A. (1973). The mind's eye in chess. In W. G. Chase (Ed.), Visual information processing (pp. 215-281). New York: Academic Press.

Chi, M.T.H., Feltovich, P.J., \& Glaser, R.(1981). Categorization and representation of physics problems by experts and novices. Cognitive Science, 5, 121-152.

Chi, M.T.H., Glaser, R., \& Rees, E. (1982). Expertise in problem solving. In R.J. Sternberg (Ed.), Advances in the psychology of human intelligence. Volume 1 (pp. 7-75). Hillsdale, $\mathrm{NJ}$ : Erlbaum

Danner, D., Hagemann, D., Holt, D.V., Bechthold, M., Schankin, A., Wüstenberg, S., \& Funke, J. (2011). Measuring performance in a Complex Problem Solving task: Reliability and validity of the Tailorshop simulation. Journal of Individual Differences, 32, 225-233.

Dienes, Z. \& Fahey, R. (1995). Role of specific instances in controlling a dynamic system. Journal of Experimental Psychology: Learning, Memory and Cognition, 21, 848-862.

Dörner, D. (1976). Problemlösen als Informationsverarbeitung [Problem solving as information processing]. Stuttgart: Kohlhammer.

Dörner, D. (1986). Diagnostik der operativen Intelligenz [Assessment of operative intelligence]. Diagnostica, 32, 4, 290-308.

Dörner, D. (1989). Die Logik des Misslingens. Strategisches Denken in komplexen Situationen [Logic of failure. Strategic thinking in complex situations]. Hamburg: Rowohlt.

Dörner, D., Kreuzig, H.W., Reither, F. \& Stäudel, T. (1983). Lohhausen: Vom Umgang mit Komplexität [Lohhausen: On handling complexity]. Bern: Huber.

Dunbar, K. (1998). Problem solving. In W. Bechtel, \& G. Graham (Eds.). A companion to 
Cognitive Science (pp. 289-298). London: Blackwell.

Duncker, K. (1945). On problem solving. Psychological Monographs, 58. American Psychological Association.

Edwards, W. (1962). Dynamic decision theory and probabilistic information processing. Human Factors, 4, 59-73.

Engelhart, M., Funke, J. \& Sager, S. (2011). A new test-scenario for optimization-based analysis and training of human decision making. Poster presented at the SIAM Conference on Optimization (SIOPT 2011), May 16-19, 2011, Darmstadtium Conference Center, Darmstadt, Germany.

Frensch, P. \& Funke, J. (1995). Complex Problem Solving: The European perspective. Hillsdale, NJ: Erlbaum.

Funke, J. (1985). Steuerung dynamischer Systeme durch Aufbau und Anwendung subjektiver Kausalmodelle [Control of dynamic systems via Construction and Application of subjective causal models]. Zeitschrift für Psychologie, 193, 443-465.

Funke, J. (1992). Wissen über dynamische Systeme: Erwerb, Repräsentation und Anwendung [Knowledge about dynamic systems: Acquisition, Representation and Application]. Heidelberg: Springer.

Funke, J. (1993). Microworlds based on linear equation systems: a new approach to Complex Problem Solving and experimental results. In G. Strube \& K.F. Wender (Eds.), The cognitive psychology of knowledge (pp. 313-330). Amsterdam: North-Holland.

Funke, J. (2001). Dynamic systems as tools for analysing human judgement. Thinking and Reasoning, 7, 69-89.

Funke, J. (2003). Problemlösendes Denken [Problem Solving Thinking]. Stuttgart: Kohlhammer.

Funke, J. (2010). Complex problem solving: A case for complex cognition? Cognitive Processing, 11, 133-142.

Funke, J. (2011). Problemlösen [Problem Solving]. In T. Betsch, J. Funke \& H. Plessner (Eds.), Denken - Urteilen Entscheiden Problemlösen (pp.135-199). Heidelberg: Springer.

Gaschler, R. (2009). Information reduction as item-general strategy change. Retrieved, July, 2011, from http://edoc.hu-berlin.de/dissertationen/gaschler-robert-2009-06-30/ PDF/gaschler.pdf

Gigerenzer, G., \& Brighton, H. (2009). Homo heuristicus: Why biased minds make better inferences. Topics in Cognitive Science, 1, 107-143.

Gonzalez, C. \& Lebiere, C. (2005). Instance-based cognitive models of decision making. In Zizzo, D. \& Courakis, A. (Eds.), Transfer of knowledge in economic decision making. New York: Palgrave McMillan.

Gonzalez, C., Lerch, F. J., \& Lebiere, C. (2003). Instance-based learning in dynamic decision making, Cognitive Science, 27, 591-635.

Greiff, S. (in press). Individualdiagnostik der Problemlösefähigkeit [Individual diagnostics of 
problem solving competency]. Münster: Waxmann. Forthcoming 2012.

Haider, H. \& Frensch, P. A. (1996). The role of information reduction in skill acquisition. Cognitive Psychology, 30, 304-337.

Halford, G.S., Wilson, W.H., \& Phillips, S. (1998). Processing capacity defined by relational complexity: Implications for comparative, developmental and cognitive psychology. Behavioral and Brain Sciences, 21, 803-865.

Horn, J. L., \& Blankson, N. (2005). Foundations for better understanding of cognitive abilities. In D. P. Flanagan, \& P. L. Harrison (Eds.), Contemporary intellectual assessment: Theories, tests, and issues (pp.41-68), 2nd ed. New York: Guilford Press.

Jäger, A.O., Süß. H.-M., \& Beauducel, A. (1997). Berliner Intelligenzstrukturtest. BIS-Test, Form 4 [Berlin Intelligence Structure test. BIS-Test, Form 4]. Göttingen: Hogrefe.

Johnson, E.J. (1988). Expertise and decision under uncertainty: Performance and process. In M.T.H. Chi, R. Glaser \& M.J. Farr (Eds.), The nature of expertise (pp.209-228). Hillsdale, NJ: Erlbaum.

Kalyuga, S. (2007). Expertise reversal effect and its implications for learner-tailored instruction. Educational Psychology Review, 19, 509-539.

Klahr, D. \& Dunbar, K. (1988). Dual space search during scientific reasoning. Cognitive Science, 12, 1-48.

Klauer, K.C., (1993). Belastung und Entlastung beim Problemlösen. Eine Theorie des deklarativen Vereinfachens [Charge and discharge in problem solving. A theory of declarative simplification]. Göttingen: Hogrefe.

Larkin, J.H. (1983). The role of problem representation in physics. In D. Gentner \& A. Collins (Eds.), Mental models (pp.75-98). Hillsdale, NJ: Erlbaum.

Lebiere, C., Wallach, D., \& Taatgen, N. (1998). Implicit and explicit learning in ACT-R. In F.E. Ritter \& R.M. Young (Eds.), Proceedings of the second European Conference on Cognitive Modelling (ECCM-98) (pp. 183-189). Nottingham, UK: Nottingham University Press.

Leutner, D. (2002). The fuzzy relationship of intelligence and problem solving in computer simulations. Computers in Human Behavior, 18, 685-697.

Leutner, D., Wirth, J., Klieme, E., \& Funke, J. (2005). Ansätze zur Operationalisierung und deren Erprobung im Feldtest zu PISA 2000 [Approaches concerning operationalization and their testing in the field test in the context of PISA 2000]. In E. Klieme, D. Leutner \& J. Wirth (Eds.), Problemlösekompetenz von Schülerinnen und Schülern (pp. 21-36). Wiesbaden: VS Verlag für Sozialwissenschaften.

Logan, G.D. (1988). Toward an instance theory of automatization. Psychological Review, 95, 492-527.

MacGregor, J. N., Ormerod, T. C., \& Chronicle, E. P. (2001). Information-processing and insight: A process model of performance on the nine-dot and related problems. Journal of Experimental Psychology: Learning, Memory and Cognition, 27, 176-201.

Newell, A. \& Simon, H.A. (1972). Human problem solving. Englewood Cliffs, NJ: Prentice-Hall. 
Novick, L.R., \& Bassok, M. (2005). Problem Solving. In Holyoak, K.J. \& Morrison, R.G. (Eds.), The Cambridge Handbook of Thinking and Reasoning (pp. 321-350). Cambridge: University Press.

Ohlsson, S. (1992). Information processing explanations of insight and related phenomena. In M.T. Keane \& K.J. Gilhooly (Eds.), Advances in the psychology of thinking. London: Harvester Wheatsheaf.

Osman, M. (2010). Controlling uncertainty: A review of human behavior in complex dynamic environments. Psychological Bulletin, 136, 65-86.

Putz-Osterloh, W. (1981). Über die Beziehung zwischen Testintelligenz und Problemlöseerfolg [On the relationship between test intelligence and problem solving performance]. Zeitschrift für Psychologie, 189, 79-100.

Quesada, J., Kintsch, W., \& Gomez, E. (2005). Complex Problem Solving: A field in search of a definition? Theoretical Issues in Ergonomic Science, 6(1), 5-33.

Rieskamp, J. \& Otto, P.E. (2006). SSL: A theory of how people learn to select strategies. Jorunal of Experimental Psychology: General, 135, 207-236.

Schoppek, W. (2002). Examples, rules, and strategies in the control of dynamic systems. Cognitive Science Quarterly, 2, 63-92.

Simon, H. A. \& Gobet, F. (1996). Templates in chess memory: A mechanism for recalling several boards. Cognitive Science, 31, 1-40.

Simon, H.A. \& Lea, G. (1974). Problem solving and rule induction: A unified view. In L.W. Greff (Ed.), Knowledge and cognition (pp. 105-127). Hillsdale, NJ: Erlbaum.

Sternberg, R.J. (1982). Reasoning, problem solving and intelligence. In R.J. Sternberg (Ed.), Handbook of human intelligence (pp. 225-307). Cambridge: University Press.

Sternberg, R.J. \& Frensch, P.A. (Eds.). (1991). Complex Problem Solving:Principles and mechanisms. Hillsdale, NJ: Erlbaum.

Strunk, G. \& Schiepek, G. (2006). Systemische Psychologie. Eine Einführung in die komplexen Grundlagen menschlichen Verhaltens [Systemic Psychology. An introduction in the complex basics of human behaviour]. München: Elsevier.

Süß, H.-M., Oberauer, K. \& Kersting, M. (1993). Intellektuelle Fähigkeiten und die Steuerung komplexer Systeme [Intellectual Abilities and the Control of complex systems]. Sprache \& Kognition, 12, 83-97.

Sweller, J. (1988). Cognitive load during problem solving: Effects on learning. Cognitive Science, 12, 257-285.

Sweller, J. (2005). Implications of cognitive load theory for multimedia learning. In R.E. Mayer (Ed.), The Cambridge handbook of multimedia learning (pp. 19-30). Cambridge: University Press.

Tschirgi, J.E. (1980). Sensible reasoning: A hypothesis about hypotheses. Child Development, 51,1-10.

Vollmeyer, R., Burns, B.D., \& Holyoak, K.J. (1996). The impact of goal specifity and systematicity of strategies on the acquisition of problem structure. Cognitive Science, 20, 70-100. 
Von Glasersfeld, E. (1997). Radikaler Konstruktivismus. Ideen, Ergebnisse, Probleme [Radical Constructivism. Ideas, Results, Problems]. Frankfurt: Suhrkamp.

Wechsler, D. (1944). The measurement of adult intelligence. Baltimore:Williams \& Wilkins.

Wenke, D., Frensch, P. A., \& Funke, J. (2005). Complex Problem Solving and intelligence: Empirical relation and causal direction. In R. J. Sternberg \& J. E. Pretz (Eds.), Cognition and intelligence: Identifying the mechanisms of the mind (pp. 160-187). New York: Cambridge University Press.

Wittmann, W.W. \& Süß, H.-M. (1999). Investigating the paths between working memory, intelligence, knowledge and Complex Problem Solving: Perfomances via Brunswiksymmetry. In P.L. Ackermann, P.C. Kyllonen \& R.D. Roberts (Eds.), Learning and individual differences: Process, trait and content (pp.77-108). Washington: American Psychological Association.

Wüstenberg, S., Greiff, S. \& Funke, J. (in press). Complex Problem Solving. More than reasoning? Intelligence. Forthcoming 2012. 